\title{
Grasp Force Estimation from HD-EMG Recordings with Channel Selection Using Elastic Nets: Preliminary Study
}

(C) 2018 IEEE. Personal use of this material is permitted. Permission from IEEE must be obtained for all other uses, in any current or future media, including reprinting/republishing this material for advertising or promotional purposes, creating new collective works, for resale or redistribution to servers or lists, or reuse of any copyrighted component of this work in other works. The DOI associated with this publication is 10.1109/BIOROB.2018.8487894. The published version is available at the following link: https://ieeexplore.ieee.org/document/8487894 


\title{
Grasp Force Estimation from HD-EMG Recordings with Channel Selection Using Elastic Nets: Preliminary Study
}

\author{
Itzel Jared Rodriguez Martinez, Francesco Clemente, Gunter Kanitz, Andrea Mannini, \\ Angelo Maria Sabatini, Senior Member, IEEE and Christian Cipriani, Senior Member, IEEE
}

\begin{abstract}
The force applied with a prosthetic device is fundamental for the correct handling of objects in daily tasks. However, it is also a factor that normally gets relegated to a secondary plane, as researchers mainly focus on decoding the users intent in terms of movements to be performed. Continuous estimates of the grasp force from the electromyographic (EMG) signals were proposed in the past. As motor actions are preplanned in humans, we hypothesized that it would be possible to decode the intended grasp force from the transient state of the EMG signal. We tested this hypothesis by using features extracted from surface HD-EMG recordings from forearm muscles, classified using artificial neural networks. Data from 6 able-bodied subjects were collected. They were trained and tested at segments of $120 \mathrm{~ms}$ with $20 \mathrm{~ms}$ overlap, starting $1 \mathrm{~s}$ before and ending $0.5 \mathrm{~s}$ after the detection of the onset with different subsets of channels. The results obtained showed that the transient phase contains information about the target grasp force, achieving predictions of $2.62 \%$ MVC average absolute errors within $430 \mathrm{~ms}$ from the onset of the EMG.
\end{abstract}

\section{INTRODUCTION}

Myoelectric prosthetic hands rely on the electromyographic (EMG) signals from the residual muscles in the stump as their control source. The common strategy used to control such devices in clinical settings is the use of the two-state amplitude modulation EMG controller proposed by Bottomley in 1960 [1]. Since then, with the emergence of multifunctional hands, control strategies based on pattern recognition have arisen in the research field, trying to differentiate between muscular patterns pertaining to different hand movements and, in turn, control multiple grasps of a dexterous prosthesis [2].

Albeit significant efforts have been put into the decoding of user motor intent, the estimation of the grasp force (GF) that should be exerted by the prosthetic hand was relegated to a secondary plane and classically set proportional to the amplitude of the steady-state of the EMG signals [3]. However, it was proven by several groups that in humans there is no linear relationship between the EMG signals and the generated GF [4]. Using intramuscular EMG recordings and non-linear methods, it was possible to relate the EMG signals of various muscles with their force output [5], [6]. Specifically, the latter was found to be modulated by the number of recruited motor

This work was partially funded by the European Commission under the DeTOP project (EIT-ICT-24-2015, GA \#687905) and partially by INAIL (the Italian national workers compensation) under the CECA2020 project. The work of C.C. was partially funded by the European Research Council under the MYKI project (ERC-2015-StG, Grant no. 679820).

All the authors are with the BioRobotics Institute, Scuola Superiore SantAnna, 56025 Pontedera, PI, Italy itzel jared.rodriguezmartinez@santannapisa.it units and their activation frequency [6][8]. Similar models were proposed for surface EMG recordings, providing a continuous muscle force estimate from the recorded EMG signals [4], [7], [8].

Both for the determination of the force and for the classification of the movement, researchers traditionally based their estimates on the steady state of EMG signals [2]. However, it was found that the temporal structure of this phase is non deterministic due to the active modification of recruitment and firing patterns needed to sustain the contraction [9]. In contrast, the transient phase, that is the phase from the onset to the stabilization of the signal, of upper arm muscles showed a deterministic structure [10] that suggest that this phase is more informative of the intention of movement of the patient. This hypothesis was tested using different classifiers for the decoding of the movement of intent with encouraging outcomes [11].

As movements and forces exerted on objects are preplanned in humans [12], we hypothesized that the transient phase of the EMG also included information on the GF that the subject wants to exert on the object. If so, it would imply that all the information needed to interpret the intention of the user can be extracted from this small portion of signal.

In this work, we present the preliminary results of a novel strategy to decode the target GF from the transient phase of surface EMG signals. To assess our hypothesis, we collected High Density EMG (HD-EMG) data from the forearm of 6 able-bodied participants while performing a grasp and lift task of an instrumented object, measuring the GF, for varying weights of the object (i.e. from $250 \mathrm{~g}$ to $1 \mathrm{~kg}$ ). In this study, with the recorded EMG signals we calculated the Mean Absolute Value (MAV) and the Waveform Length (WL) features with a window length of $120 \mathrm{~ms}$ (using the sliding windows method), split the trials into segments of $120 \mathrm{~ms}$ and selected the best subsets of channels with elastic nets to be used as input of an artificial neural network (ANN) for each segment. Our results show that the error in predicting the GF decreases right after the onset of the EMG, suggesting that the transient phase of EMG signals indeed contains information to decode the target GF.

\section{MATERIALS AND METHODS}

Six able-bodied participants (aged $28 \pm 3.29$ years, righthanded, three males) without any known neuromuscular disorder took part in this study. The subjects seated on a chair with a straight back, the joint of the right elbow higher 
than the wrist and their hand resting on a starting position on the table (Fig. 1).

An instrumented object (IO) was placed $15 \mathrm{~cm}$ in front of the starting position, with the grasp site placed at the same height as the hand. The size of the IO at the grasp site was $6 \times 2.2 \times 5 \mathrm{~cm}$ height, width and depth respectively $(17.8 \times 6.8 \times 6 \mathrm{~cm}$ overall $)$ and contained a 6 axis load cell (NANO17, ATI Industrial Automation Inc.) to measure the GF. The load force was measured by a piezo-resistive force sensor (FSG series, Honeywell Sensotec) integrated into a scale under the IO. A monitor placed in the line of sight of the participant, provided instructions about the experiment. Hand movements performed by the subject were recorded with a data-glove (18 sensors CyberGlove III by Cyber Glove Systems LLC, San Jose, CA). EMG signals were recorded using an EMG amplifier (EMG-USB2+, OT Bioelettronica, Turin, Italy) with three 64 contact $(8 \times 8)$ electrode arrays with $10 \mathrm{~mm}$ inter-electrode distance (ELSCH064NM3, Spes Medica srl, Genova, Italy). Two of the three electrode arrays were centered on the extensor digitorum (ED) and the flexor digitorum (FD) muscles controlling the hand aperture during prehension and the third electrode array was placed on the thumb flexor (i.e. flexor pollicis longus FPL) for its contribution to the GF. The EMG data were recorded with a sample frequency of $10.24 \mathrm{kHz}$ with a low-pass filter at $4.4 \mathrm{kHz}$ and a high-pass filter at $10 \mathrm{~Hz}$. The lift off/return event of the hand, indicating the beginning and end of each trial, was detected with an FRS sensor placed in the starting position.

Subjects were asked to always maintain the same relaxed three-digital grasp posture at the starting position. They were instructed to, following a visual cue provided by the monitor: (i) move their right arm to reach the IO, (ii) grasp it using a three-digit grasp, (iii) lift it by $10 \mathrm{~cm}$ (iv) and hold it for $2 \mathrm{~s}$ before (v) placing it back on the table and (vi) returning their hand to the starting position. This procedure was repeated changing the weight of the IO from $250 \mathrm{~g}$ to $1000 \mathrm{~g}$ in steps of $250 \mathrm{~g}$. Each weight was lifted 15 times for each series and 5 series were executed by each subject, for a total of $(15$ trials $\times 5$ series $\times 4$ weights $=) 300$ trials. In between series subjects were given time to rest. The order of the weights was randomized among subjects.

At the beginning and the end of the experiment, in order to determine their maximum voluntary contraction (MVC), each subject was asked to grasp and hold the IO for $\sim 3$ s by applying the maximum GF three times [13]. Of the six repetitions, the grasp with the highest force was used to determine the MVC.

\section{DATA ANALYSIS}

Offline analyses were performed using MATLAB 2016b software (The Mathworks, Natick, MA, USA). The data were down-sampled to $2 \mathrm{kHz}$ and, of the 15 lifts of each series, the first three were disregarded for the analysis in order to discard data variability due to the subjects adapting to the new weight condition [14]. On the remaining $(12$ trials $\times 5$ series $\times 4$ weights $=) 240$ trials the onset of the

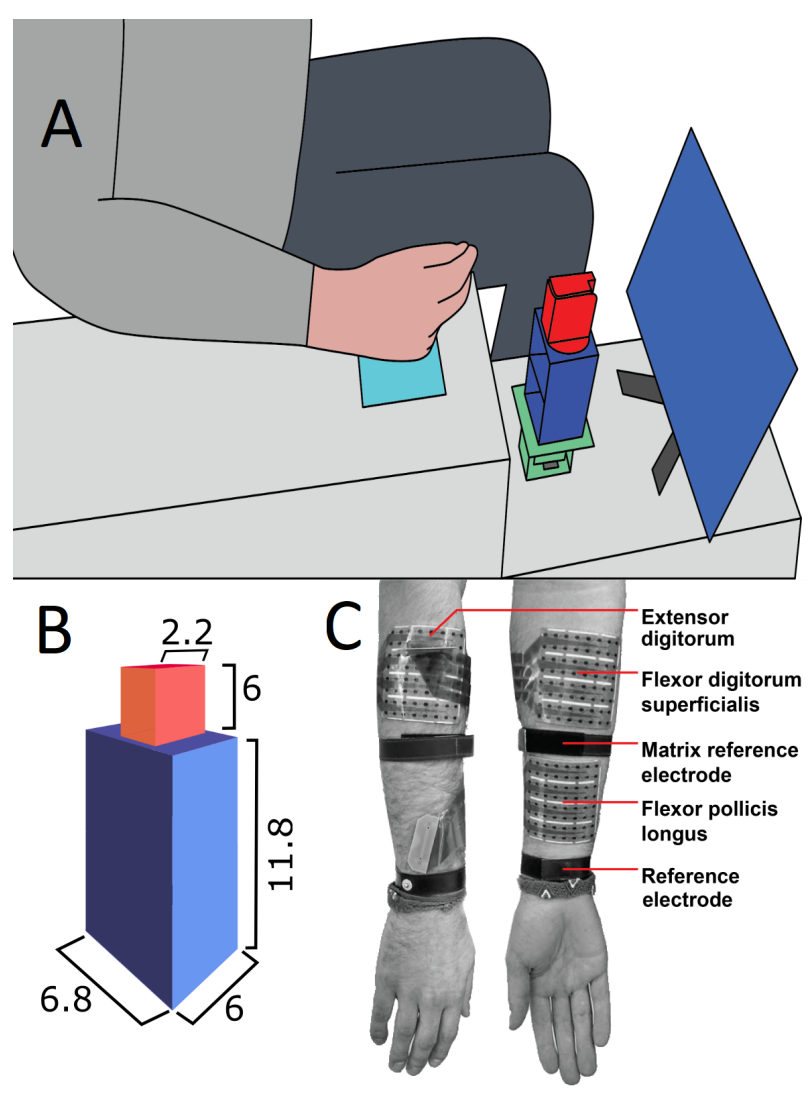

Fig. 1. Experimental setup. A) Subject seated with the right hand resting on the lift off/return event sensor (in light blue) and the instrumented object and monitor in line of sight. A load force sensor (green) was placed underneath the instrumented object, which consisted of a box for placing different weights (dark blue) and a GF sensor (red). B) Instrumented object dimensions (in $\mathrm{cm}$ ). C) Placement of HD-EMG electrodes with indicated target muscles and reference electrodes.

grasp was defined as the contact event between the subject and the IO. This was detected by setting a threshold on the GF equal to three standard deviations of the GF measured during the reaching phase. The target GF provided to the ANN was determined by calculating the mean of the plateau of the GF (i.e. the GF from $400 \mathrm{~ms}$ after the onset until the end of the lift, Fig. 2) normalized by the GF value obtained during MVC. For the rest of the analysis, the trials were segmented from $1 \mathrm{~s}$ before to $0.5 \mathrm{~s}$ after the onset in order to include data from the reaching phase, the transient EMG and the beginning of the EMG steady state.

The EMG signals were filtered with a $4^{\text {th }}$ order bandpass Butterworth filter with cut-off frequencies at $20 \mathrm{~Hz}$ and $500 \mathrm{~Hz}$. The $8 \times 8$ monopolar EMG signals of each matrix of electrodes were converted into $7 \times 8$ bipolar signals by differentiating neighbouring electrodes in the direction of the muscle fibers. These signals were then normalized by the MVC for each subject to have an estimate of muscle activation. From the filtered data, the MAV and the WL features were extracted using a sliding window with a step of $10 \mathrm{~ms}$ and a window length $w_{L}$ of $120 \mathrm{~ms}$. The resulting data were split into time segments using sliding windows without overlap with length $w_{T}=120 \mathrm{~ms}$ to have representative 


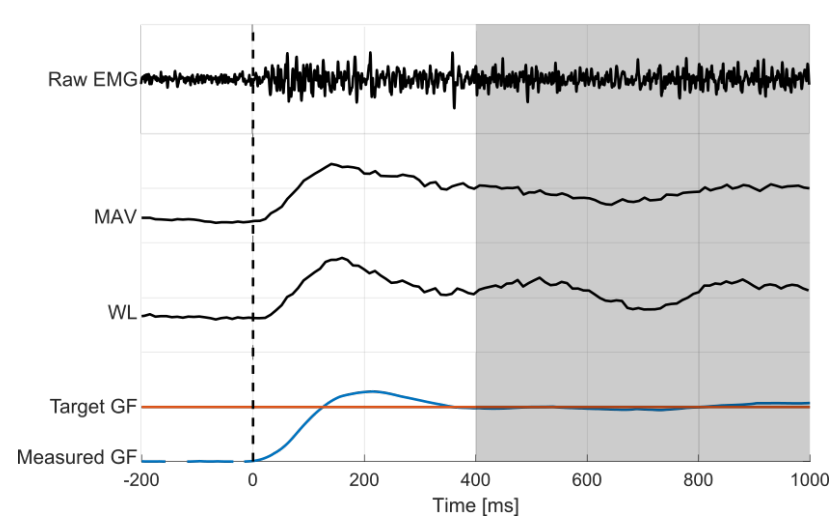

Fig. 2. Representative time series of the EMG (raw and processed), and the Grip Force. The lower plot represents the extraction of the target GF (red) for the ANN training from the measured GF (blue) during a representative trial. Trials are synchronized at the onset of the EMG (dotted black line). The gray area indicates the part of the trial averaged to obtain the target GF from the measured GF.

samples of the trials.

The rest of the analysis was performed independently for each of the six configurations (i.e. two features and three sets of channels) and time segments. The 240 lifts were split into train and test set and the selection of the best sets containing $n_{C H} \in[4,8,16]$ channels was performed by tuning the lambda parameter of an elastic nets analysis (alpha $=0.5)$ on the train set [15]. The best sets were used as input of an ANN with three hidden layers and the target GF as target output. Each of the layers of the ANN contained three neurons with rectified linear unit activation functions and batch normalization. The quality of the ANN fitting was evaluated through the absolute error between predicted and target GF. To select the best proportion for the train and test set, a preliminary analysis was performed for the configuration of eight channels by calculating the learning curves with the proportions of train/test $\in[20 / 80,40 / 60$, $60 / 40,80 / 20$ ] $\%$ of the trials [16].

For the statistical analysis of the errors, a two-way ANOVA (factors: features and number of channels) with post hoc pair-wise comparisons using Tukeys honestly significant difference test was computed. A significance level of $p=0.05$ was used for the ANOVA. From the results, one configuration was selected for the posterior analysis. With this configuration a threshold on the absolute error was set from the distribution of the mean absolute errors for each weight per subject. Then, for each trial, the time delay from the onset when the error crossed that threshold and the absolute error obtained $150 \mathrm{~ms}$ after the onset were determined

\section{RESULTS AND DISCUSSION}

For the selection of the proportion of the train/test sets, the mean absolute error for each proportion and time segment was calculated and the learning curve evaluated for the 8 channels configuration (Fig. 3). For both features, changing the proportion of the train/test sets did not seem to have a significant impact on the performance. Therefore, for the rest of the analysis we selected the proportion with the minimum

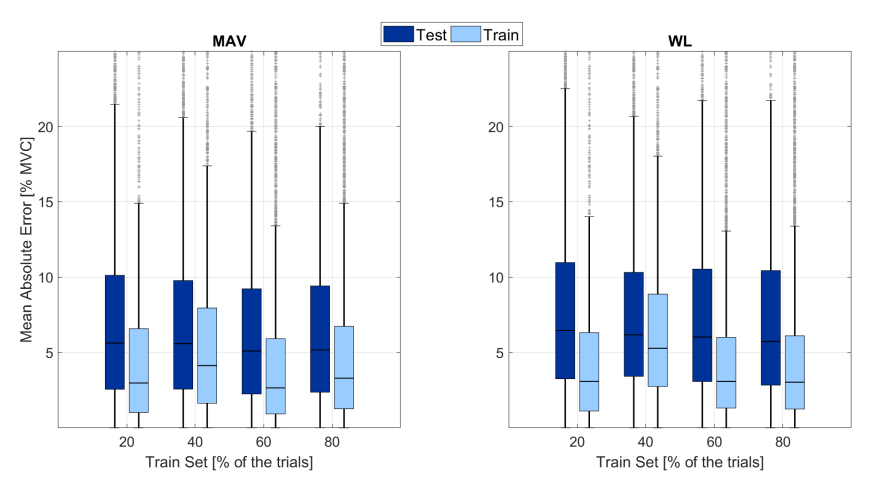

Fig. 3. Selection of the train/test dataset proportion on 8 channels. The vertical axis represents the difference between the mean absolute errors of the train and test set. The horizontal lines within each boxplot represent the median across time segments, subjects and trials.

difference between the median errors of the train and test sets, that is, $40 \%$ of the trials for train and $60 \%$ for test.

The selection of the best subsets of channels was performed using elastic nets from the training data (Fig. 4). The reduction of channels was performed because, for clinical and long term applications, it is not practical to implement a large number of electrodes on a prosthetic device. However, the selection of the best locations for electrode placement is not trivial and usually is determined by a trained prosthetist specifically for each subject. This personalisation is due to the physiological differences among subjects, which are particularly large in amputees. Therefore, our approach was to use the HD-EMG arrays for the recording and let the algorithm select the best channels for a later fixed implementation into the final prosthesis socket. The four channels configuration was selected because it represents a (more) clinically viable solution and its performance was meant to be tested against that of a larger number of electrodes.
FPL

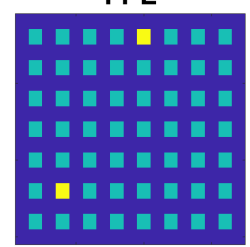

FD

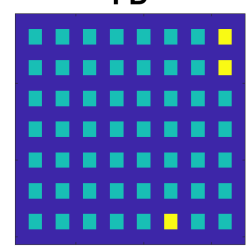

ED

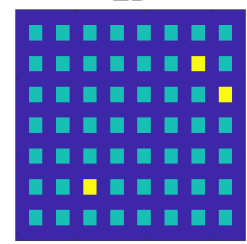

Fig. 4. Representative sample of the channels selected from each electrode array. It corresponds to the selection for one subject with the MAV feature and 8 channels at the last time segment. The blue grid represents the area of the electrode array, the yellow squares the selected channels and the greens the rest of the channels.

For the estimation of the target GF, an ANN for each configuration and segment was trained and tested and the absolute error between the predicted and the target GF was calculated. The absolute error was found to be more or less constant at the random guess value (mean absolute error of $6.98 \%$ MVC across subjects) during the reaching phase and started to decrease right after the onset (Fig. 5). The mean absolute errors obtained during the reaching phase, the minimum mean absolute errors obtained and the time 


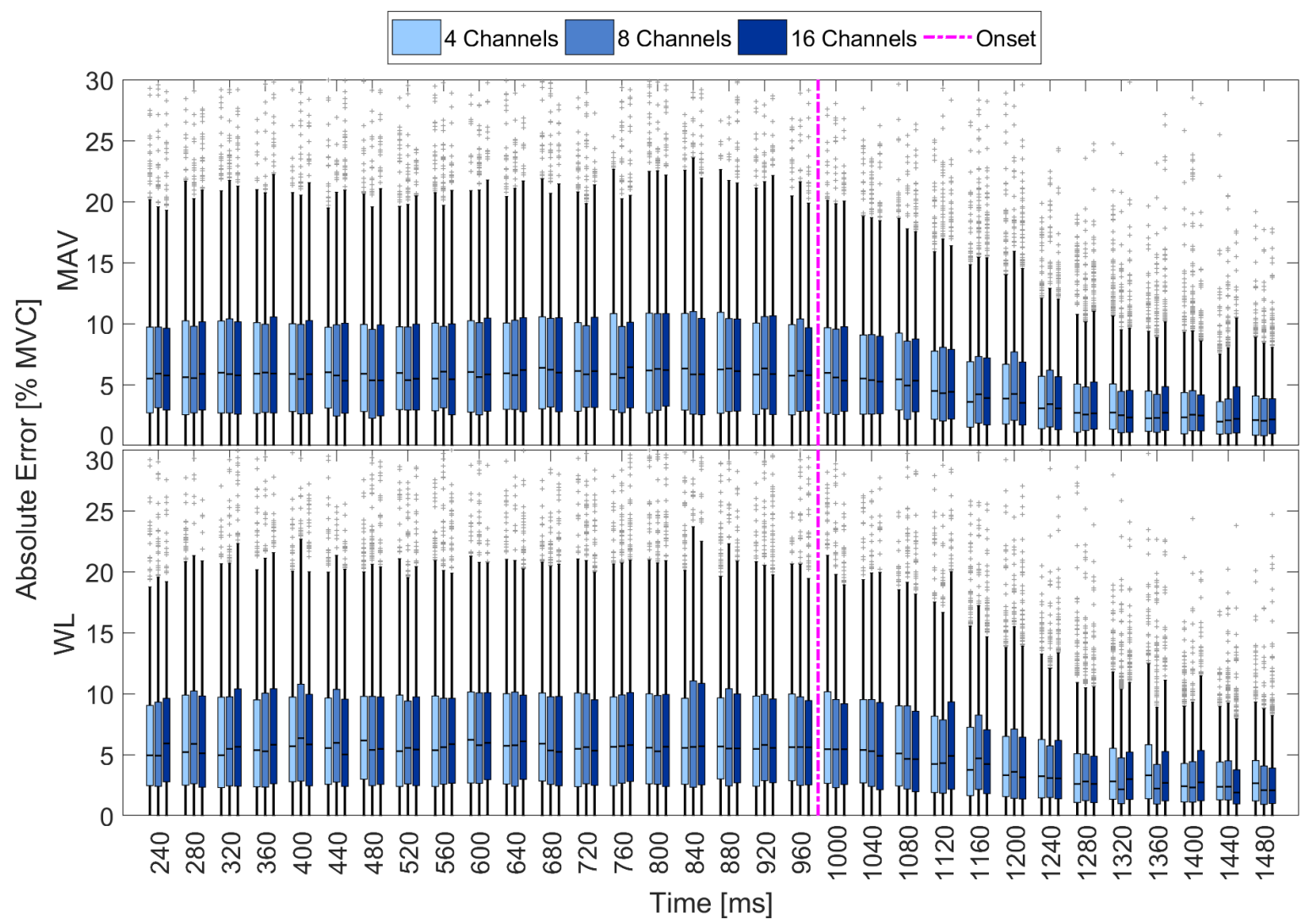

Fig. 5. Absolute errors between the predicted and the target GF obtained with $w_{L}$ and $w_{T}$ of $120 \mathrm{~ms}$. The dotted blue line represents the moment of the onset detection, the light blue boxplots the errors for the configuration with 4 channels, the medium blue with 8 and the dark blue with 16 . The circle within each boxplot represents the median, the bottom and top edges of the box indicate respectively the $25^{\text {th }}$ and $75^{\text {th }}$ percentiles, the whiskers the standard deviation and the ' + ' the outliers. The errors are reported as a percentage of the MVC.

after the onset at which the minimum was obtained are shown in Table 1. The minimum error (between 2.62 and $2.84 \%$ MVC) with all the configurations was obtained between 430 and $470 \mathrm{~ms}$, achieving an improvement between 4.17 and $4.66 \% \mathrm{MVC}$ with respect to the maximum error.

TABLE I

REACHING PHASE ERROR, MINIMUM ERROR AND TIMING OF THE MINIMUM ERROR.

\begin{tabular}{|l|c|c|c|c|c|c|}
\hline & \multicolumn{2}{|c|}{ 4 Channels } & \multicolumn{2}{c|}{ 8 Channels } & \multicolumn{2}{c|}{ 16 Channels } \\
\cline { 2 - 7 } & MAV & WL & MAV & WL & MAV & WL \\
\hline $\begin{array}{l}\text { Mean Absolute } \\
\text { Error Reaching } \\
\text { Phase (\%MVC) }\end{array}$ & 7.28 & 7.02 & 7.19 & 7.01 & 7.23 & 6.97 \\
\hline $\begin{array}{l}\text { Minimum Mean } \\
\text { Absolute Error } \\
\text { (\%MVC) }\end{array}$ & 2.62 & 2.71 & 2.66 & 2.84 & 2.77 & 2.73 \\
\hline $\begin{array}{l}\text { Time after the } \\
\text { onset of the mini- } \\
\text { mum error (ms) }\end{array}$ & 430 & 450 & 470 & 470 & 450 & 450 \\
\hline
\end{tabular}

In order to determine if the differences found among configurations were significant, ANOVA and Tukey-s post/hoc comparison tests were performed (Fig. 6) using the mean absolute errors among trials of each subject. The two-way ANOVA reported that the number of channels $(\mathrm{F}(2,2267)=0.09, \mathrm{p}=0.917)$ and features $(\mathrm{F}(1,2267)=2.12$, $\mathrm{p}=0.1452$ ) were not significant. Therefore, the configuration of WL and 16 channels was used for the selection of the threshold on the absolute error. From the distribution of the mean absolute error for each weight per subject (Fig. 7) the set threshold was of $3 \% \mathrm{MVC}$. Then, for each trial, the time delay from the onset when the error crossed that threshold and the absolute error obtained $150 \mathrm{~ms}$ after the onset were determined. Of the 216 trials per weight (all the subjects), in $8.8 \%$ the error did not cross the set threshold. The remaining trials crossed the threshold on average $170 \mathrm{~ms}$ after the onset (Fig. 8) and kept improving. In addition, they got an error of $4.3 \% \mathrm{MVC}$ in average $150 \mathrm{~ms}$ after the onset (the transient lasted roughly 200 380 ms). This confirms our hypothesis that the transient phase of the EMG signal contains useful information to decode the target GF.

Since this was a pilot study, the $w_{L}$ and $w_{T}$ were restricted to one window of $120 \mathrm{~ms}$ because then, the transient could be enclosed in $10 \sim 19$ time segments and it would provide the ANN with a significant amount of data across time, without exceeding the optimal controller delay for myoelectric prostheses (between 100 and $125 \mathrm{~ms}$ ) [17]. As for the weights used for the lifts, other studies target percentages of the MVC [5], [18], however, since a prosthetic hand is mainly 
used to grasp and lift objects in daily tasks, precise GFs are required. The patterns responsible for the generation of such complex muscle activity are developed from experience and tuned by the physical properties, like weight or shape, of the object to manipulate [12]. This implies that for tasks of grasp and lift, predefined internal motor models exist and are adjusted accordingly by previous experiences and the visual and haptic feedback from the target object, providing specific motor commands prior to the execution of the action. These models allow to manipulate each object with the proper GF, consistent with its weight and physical characteristics. Therefore, to produce specific GFs and the associated EMG contractions, weights within the range of those manipulated in daily tasks were selected.
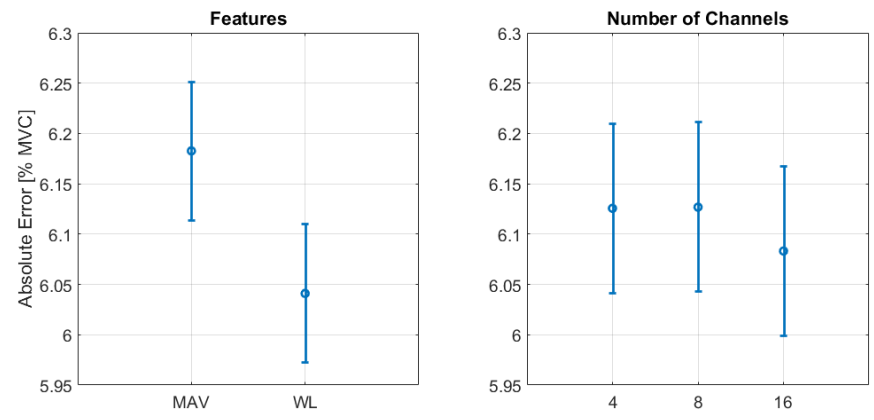

Fig. 6. Statistical analysis of the absolute errors. Right: With the features as factor. Left: With the number of channels as factor, the dotted magenta line represents the onset. The intervals correspond to the standard errors and the circles to the mean.

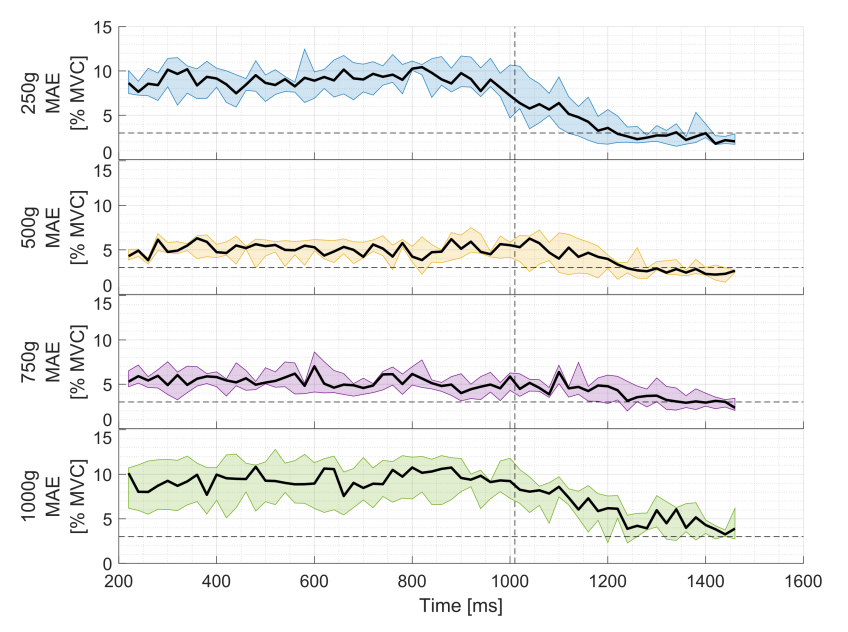

Fig. 7. Absolute errors per weight between the predicted and the target GF obtained with the feature WL, 16 channels and $w_{L}$ and $w_{T}$ of $120 \mathrm{~ms}$. The dotted vertical line represents the onset, the black line the median, the colored area the interquartile range and the dotter horizontal line the set threshold.

Our results fall within the same range of error of previous studies about the decoding of the force from EMG signals where the best estimates obtained were mean absolute errors of 4.21 and $9.2 \% \mathrm{MVC}$ on the steady state[19], [20]. However, the comparison with previous studies is limited due to the difference between the targeted muscles, the tasks

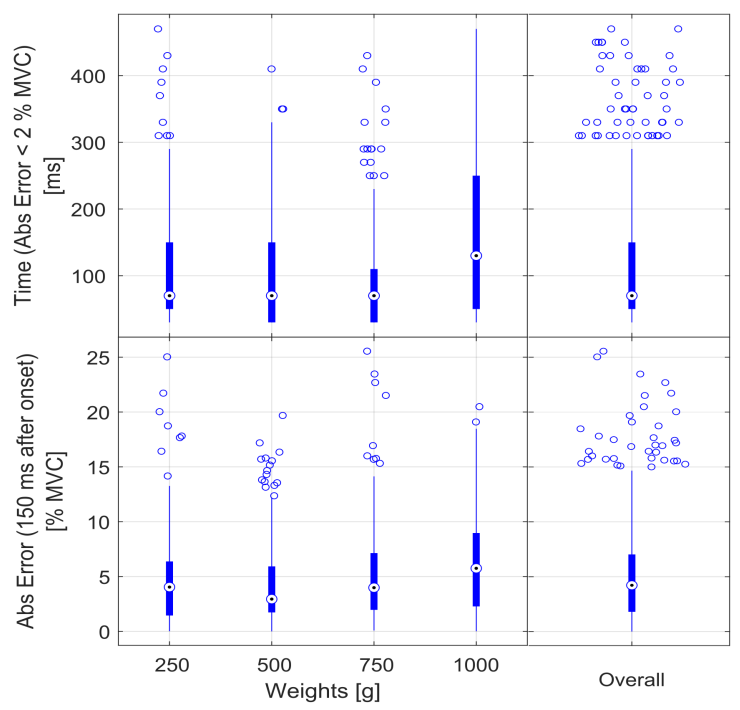

Fig. 8. Time and error obtained with thresholds. Top: time after the onset needed to attain an absolute error inferior to $3 \%$ MVC. Bottom: absolute Error obtained $150 \mathrm{~ms}$ after the onset. The circle within each boxplot represents the median, the bottom and top edges of the box indicate respectively the 25 th and 75 th percentiles, the whiskers the standard deviation and the ' + ' the outliers. The errors are reported as a percentage of the MVC.

performed and even the metrics used to report the results (mean absolute value, root mean square [18] and $R^{2}$ [7] being the more common).

Albeit a small improvement in performance was observed by increasing the number of channels, the statistical analysis did not show significant differences. This can be due to the nature of surface EMG signals which are affected by involuntary factors such as crosstalk from adjacent muscles, changes in the motor units discharge synchronization, individual muscle fiber potential and fatigue [21]. This leads the EMG signals to be highly correlated, prompt to noise and to the need for substantial processing when working with them. We tried to mitigate this effect by performing the channel selection using elastic nets, which is a variation of the LASSO (Least Absolute Shrinkage and Selection Operator) and the Ridge regression [15], especially designed to deal with correlated variables. However, the correlation between channels was still high (the mean correlation for 16 channels was 0.67). Moreover, another factor limiting the performance improvement is probably the fact that, in this pilot study, the optimization of the train/test set ratio was performed only on the 8 channels configuration. It would be interesting in a future work to compare the performance of the system optimizing the train/test set ratio for each configuration specifically.

Albeit present in the experimental setup, the data-glove was not used in this particular study and its data could be useful in future studies. We argue that the friction with the IO due to the glove, affected all tested configurations in a similar manner and as such did not impact the obtained results. 


\section{CONCLUSIONS}

The objective of this pilot study was to assess if the target GF could be decoded from the transient of EMG signals. For it, experimental data from 6 healthy subjects during grasp and lift tasks of objects with varying weights were collected. Then, ANNs were implemented and tested with six configurations (i.e. two features, three sets of channels one $w_{L}$ and one $w_{T}$ ) on different time segments of the lifts. The results confirmed that the transient phase contains information about the target GF. In the future, we will increase the tested configurations and tune the parameters of the ANN to obtain the best estimate of the target GF, considering not only the lowest error but also the time of response.

\section{REFERENCES}

[1] A. H. Bottomley, Myo-electric control of powered prostheses., J. Bone Joint Surg. Br., vol. 47, pp. 4115, Aug. 1965.

[2] K. B. Englehart and B. S. Hudgins, A robust, real-time control scheme for multifunction myoelectric control., IEEE Trans. Biomed. Eng., vol. 50, no. 7, pp. 84854, Jul. 2003.

[3] E. J. Scheme, B. A. Lock, L. J. Hargrove, W. Hill, U. Kuruganti, and K. Englehart, Motion normalized proportional control for improved pattern recognition-based myoelectric control, IEEE Trans. Neural Syst. Rehabil. Eng., vol. 22, no. 1, pp. 149157, 2014.

[4] D. Staudenmann, K. Roeleveld, D. F. Stegeman, and J. H. Van Dien, Methodological aspects of SEMG recordings for force estimation A tutorial and review, Journal of Electromyography and Kinesiology, vol. 20, no. 3. pp. 375387, Jun-2010.

[5] E. N. Kamavuako, K. B. Englehart, W. Jensen, and D. Farina, Simultaneous and proportional force estimation in multiple degrees of freedom from intramuscular EMG, IEEE Trans. Biomed. Eng., vol. 59, no. 7, pp. 18041807, 2012.

[6] M. F. Bg, E. Erkocevic, M. J. Niemeier, J. R. Mathiesen, A. Smidstrup, and E. N. Kamavuako, Investigation of the linear relationship between grasping force and features of intramuscular EMG, in IFMBE Proceedings, 2011, vol. 34 IFMBE, pp. 121124.

[7] J. L. G. Nielsen, S. Holmgaard, N. Jiang, K. B. Englehart, D. Farina, and P. A. Parker, Simultaneous and proportional force estimation for multifunction myoelectric prostheses using mirrored bilateral training, IEEE Trans. Biomed. Eng., vol. 58, no. 3 PART 1, pp. 681688, 2011.

[8] C. Castellini, E. Gruppioni, A. Davalli, and G. Sandini, Fine detection of grasp force and posture by amputees via surface electromyography, J. Physiol., vol. 103, no. 35, pp. 255262, May 2009.

[9] C. J. De Luca, Physiology and mathematics of myoelectric signals, IEEE Trans. Biomed. Eng., vol. 26, no. 6, pp. 313325, 1979.

[10] G. Kanitz, C. Cipriani, and B. B. Edin, Classification of transient myoelectric signals for the control of multi-grasp hand prostheses, IEEE Trans. Neural Syst. Rehabil. Eng. [Submitted]

[11] N. Jiang, T. Lorrain, and D. Farina, A state-based, proportional myoelectric control method: online validation and comparison with the clinical state-of-the-art, J. Neuroeng. Rehabil., vol. 11, no. 1, p. 110, Jul. 2014.

[12] R. S. Johansson, Sensory input and control of grip., Novartis Found. Symp., vol. 218, pp. 45-59; discussion 59-63, 1998.

[13] C. J. De Luca and E. C. Hostage, Relationship Between Firing Rate and Recruitment Threshold of Motoneurons in Voluntary Isometric Contractions, J. Neurophysiol., vol. 104, no. 2, pp. 10341046, 2010.

[14] R. S. Johansson and K. J. Cole, Sensory-motor coordination during grasping and manipulative actions, Curr. Opin. Neurobiol., vol. 2, no. 6, pp. 815823, Dec. 1992.

[15] H. Zou and T. Hastie, Regularization and variable selection via the elastic-net, J. R. Stat. Soc., vol. 67, no. 2, pp. 301320, 2005.

[16] A. K. Jain, R. P. W. W. Duin, and J. Mao, Statistical pattern recognition: a review, IEEE Trans. Pattern Anal. Mach. Intell., vol. 22, no. 1, pp. 437, 2000.

[17] T. R. Farrell and R. F. Weir, The optimal controller delay for myoelectric prostheses, IEEE Trans. Neural Syst. Rehabil. Eng., 2007.
[18] E. A. Clancy, C. Martinez-Luna, M. Wartenberg, C. Dai, and T. R. Farrell, Two degrees of freedom quasi-static EMG-force at the wrist using a minimum number of electrodes, J. Electromyogr. Kinesiol., vol. 34, pp. 2436, 2017.

[19] P. Liu, F. Martel, D. Rancourt, E. A. Clancy, and D. R. Brown, Fingertip force estimation from forearm muscle electrical activity, ICASSP, IEEE Int. Conf. Acoust. Speech Signal Process. - Proc., pp. 20692073, 2014.

[20] J. R. Potvin, R. W. Norman, and S. M. Mcgill, Mechanically corrected EMG for the continuous estimation of erector spinae muscle loading during repetitive lifting, Eur J Appl Physiol, vol. 74, pp. 119132, 1996.

[21] C. J. De Luca and R. Merletti, Surface myoelectric signal cross-talk among muscles of the leg, Electroencephalogr. Clin. Neurophysiol., vol. 69 , no. 6 , pp. $568575,1988$. 